\title{
ADMISSIBILITY OF A WIDE CLUSTER SOLUTION IN "ANISOTROPIC" HIGHER-ORDER TRAFFIC FLOW MODELS*
}

\author{
RUI-YUE XU ${ }^{\dagger}$, PENG ZHANG ${ }^{\ddagger}$, SHI-QIANG DAI ${ }^{\dagger}$, AND S. C. WONG§
}

\begin{abstract}
We analytically investigate a wide cluster solution and show that it is not admitted in some of the traffic flow models in the literature. For those traffic flow models that admit the wide cluster solution, the relationship between two important control parameters and the critical densities that divide an equilibrium solution into stable and unstable regions is thoroughly discussed in detail. We find that such wide clusters exist with a free traffic density in an unstable region, and with one or three critical densities. These results are different from the cases in the well-known higher-order traffic flow models of Payne and Whitham [H. J. Payne, "Models of freeway traffic and control," in Mathematical Models of Public Systems, A. G. Bekey, ed., Simulation Council Proc. Ser. 1, La Jolla, CA, 1971, pp. 51-61], [G. B. Whitham, Linear and Nonlinear Waves, John Wiley and Sons, New York, 1974], Kühne [R. D. Kühne, "Macroscopic freeway model for dense traffic-stop-start waves and incident detection," in Proceedings of the 9th International Symposium on Transportation and Traffic Theory, J. Volmuller and R. Hamerslag, eds., VNU Science Press, Utrecht, 1984, pp. 21-42], and Kerner and Konhäuser [B. S. Kerner and P. Konhäuser, Phys. Rev. E (3), 50 (1994), pp. 54-83].
\end{abstract}

Key words. Aw and Rascle model, hyperbolic conservation law, wide cluster, shock wave, Rankine-Hugoniot condition

AMS subject classifications. 15A15, 15A09, 15A23

DOI. $10.1137 / 06066641 \mathrm{X}$

1. Introduction. Lighthill and Whitham [14] and Richards [17] independently proposed a hydrodynamic approach to study the traffic flow problems on a homogeneous highway, which is known in the literature as the first-order LWR model. The model has recently been extended to multilane [4] and multiclass models [3, 9, 10, $25,31,30,32]$. To embody the important nonlinear phenomena in traffic flow problems, some classical higher-order traffic flow models were developed [13, 12, 15, 23] and are characterized by their capability to reproduce the stop-and-go waves that are frequently observed on the highways. The formation of clusters is related to the instability of congested traffic, of which the development into free traffic and jams is typical of phase transitions and hystereses [12, 29, 28]. However, Daganzo [5] criticized the "isotropic" nature of these models, following which a stream of so-called anisotropic higher-order models $[1,8,11,19,26,27]$ was developed.

Recently, an analytical tool that is based on the weak solution theory was proposed to give a full and concise description of a wide cluster solution in higher-order models. Zhang, Wong, and Dai [33] used the model proposed by Jiang, Wu, and

*Received by the editors July 31, 2006; accepted for publication (in revised form) August 13, 2007; published electronically December 5, 2007. This work was jointly supported by the National Natural Science Foundation of China (grants 70629001, 10532060), the National Basic Research Program of China (grant 2006CB705503), the Research Grants Council of the Hong Kong Special Administrative Region, China (project HKU7187/05E), the Shanghai Leading Academic Discipline Project (grant Y0103), and the University of Hong Kong (grant 10207394).

http://www.siam.org/journals/siap/68-2/66641.html

†Shanghai Institute of Applied Mathematics and Mechanics, Shanghai University, Shanghai 200072, People's Republic of China (monkeyxu1983@163.com, sqdai@126.com).

${ }^{\ddagger}$ Corresponding author. Shanghai Institute of Applied Mathematics and Mechanics, Shanghai University, Shanghai 200072, People's Republic of China (pengzhang@ustc.edu).

$\S$ Department of Civil Engineering, The University of Hong Kong, Pokfulam Road, Hong Kong SAR, People's Republic of China (hhecwsc@hkucc.hku.hk). 
Zhu [11] to demonstrate the solution procedure. Zhang and Wong [28] further showed that two conservation forms of the Payne-Whitham (PW) models [15, 23] have different cluster solutions that are asymptotic to the solutions of the Kühne [13] and Kerner and Konhäuser (KK) [12] models.

In this paper, we thoroughly investigate the admissibility of a wide cluster solution for several aforementioned "anisotropic" models, using the technique that was developed in $[33,28]$. The model equations are introduced in section 2 . In section 3 , the procedure for solving a wide cluster is briefly discussed. While a criterion is prescribed as an essential condition, the formulations in $[26,27]$ are excluded from further consideration because they do not admit a wide cluster solution (see section 3.1). Here, we note that cluster solutions were also derived in Greenberg [6], Siebel and Mauser [20], and Wilson and Berg [24]. With two control parameters in the "pressure" $p(\rho)=\alpha\left(\rho / \rho_{m}\right)^{\gamma}$ of Aw and Rascle [1], the admissible regions of $(\gamma, \alpha)$ for the wide cluster solution are displayed. The regions of $(\gamma, \alpha)$ that are related to the stability of an equilibrium solution are also discussed (see section 3.2). It is novel to find that the free traffic flow (the constant flow with the minimal density) of a wide cluster solution is not stable in numerous regions, whereas the jam (the flow with the maximal density in the cluster) together with the whole solution is always stable. In some of these regions, it is evident that a wide cluster solution is admitted with one or three critical densities, which are used to divide equilibrium flows into stable and unstable regions. The results from numerical simulations (see section 4) are in good agreement with all analytical findings.

2. Model equations. In macroscopic descriptions, vehicles on a highway are analogous to flows in compressible hydrodynamics $[14,17]$. We have, by the mass conservation,

$$
\rho_{t}+q_{x}=0
$$

where $\rho(x, t)$ is the density, $q(x, t)$ is the flow, and no off- or on-ramp flows are considered along the highway. By defining an average speed $v=q / \rho$, the acceleration is considered in higher-order models. We write the acceleration of the discussed "anisotropic" models in the following mutual form:

$$
v_{t}+v v_{x}=\frac{v_{e}(\rho)-v}{\tau}+\rho p^{\prime}(\rho) v_{x}
$$

where $p(\rho)$ is the "pressure" with $p^{\prime}(\rho)>0$, and $v_{e}(\rho)$ is the equilibrium speed-density relationship with $v_{e}^{\prime}(\rho)<0$. For the derivation of a stable wide cluster, we assume a nonconvex fundamental diagram $q_{e}(\rho)=\rho v_{e}(\rho)$. Precisely, $q_{e}^{\prime \prime}(\rho)<0$ for $0 \leq \rho<\rho_{I}$, and $q_{e}^{\prime \prime}(\rho)>0$ for $\rho_{I}<\rho \leq \rho_{m}$, where $q_{e}^{\prime \prime}\left(\rho_{I}\right)=0$, and $\rho_{m}$ is the jam (maximum) density. Furthermore, we assume that $\tau>\tau_{0}$ for some fixed $\tau_{0}>0$, and thus the relaxation term is bounded. Nevertheless, this model is asymptotic to the LWR model $[14,17]$ if we allow $\tau \rightarrow 0$, which leads to $v \rightarrow v_{e}(\rho)$; see [16].

It is easy to derive the two characteristic speeds of system (1)-(2): $\lambda_{1}=v-\rho p^{\prime}(\rho)$ and $\lambda_{2}=v$, which are no greater than the motion speed. Therefore, a perturbation propagates only upstream with respect to the perturbed moving vehicle. This is why the formulation is classified as "anisotropic." In contrast, the two characteristic speeds in the classical models are, respectively, smaller and greater than the traffic speed $v$, meaning that the perturbation propagates both upstream and downstream.

Several choices of the function $p(\rho)$ are listed in Table 1, corresponding to different 
TABLE 1

"Anisotropic" formulations of higher-order traffic flow models, which are distinguished by the "pressure" $p(\rho)$ or the sound speed $c(\rho)=\rho p^{\prime}(\rho)$, where $T(\rho)=t_{r}[1+E /(1+\rho)]$ and constants $c_{0}, E, t_{r}>0$.

\begin{tabular}{lll}
\hline Models & $c(\rho)$ & $p(\rho)$ \\
\hline Aw and Rascle [1] & $\rho p^{\prime}(\rho)$ & $p(\rho)$ \\
Jiang, Wu, and Zhu [11] & $c_{0}$ & $c_{0} \ln \rho$ \\
Zhang [27] & $-\rho v_{e}^{\prime}(\rho)$ & $-v_{e}(\rho)$ \\
Xue and Dai [26] & $-\rho \frac{t_{r}}{T(\rho)} v_{e}^{\prime}(\rho)$ & $-\frac{t_{r}}{T(\rho)} v_{e}(\rho)$ \\
\hline
\end{tabular}

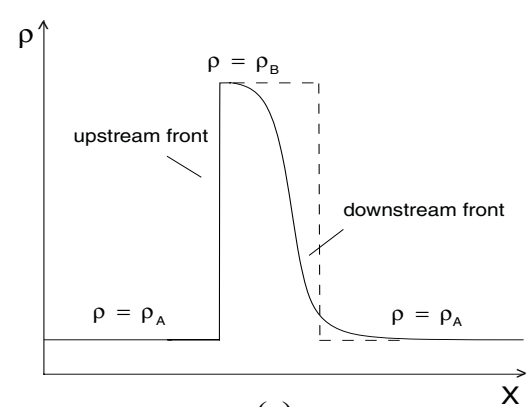

(a)

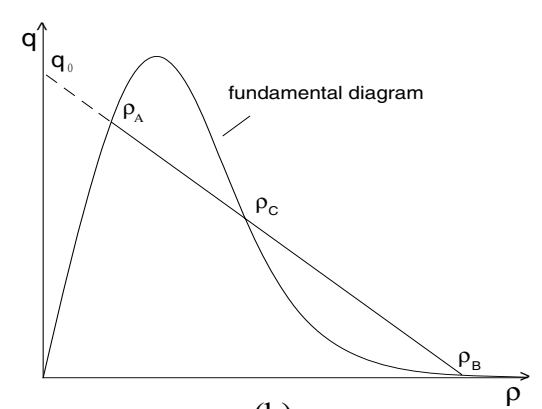

(b)

FIG. 1. Illustration of a wide cluster solution: (a) profile of the density distribution; (b) phase plot $q=q(\rho)$ in comparison to the fundamental diagram $q=q_{e}(\rho)$.

formulations in $[1,11,26,27]$. We note that Aw and Rascle [1] contained a more general model by assuming an increasing function $p(\rho)$, and that the relaxation term was considered in [16] as an improvement; see also $[2,7]$ for further development of the Aw and Rascle model. Similarly, we add such a term to the formulation of Zhang [27]; without the term, it is unlikely to derive a wide cluster solution.

3. Admissibility of a wide cluster solution. The profile of a wide cluster solution is shown in Figure 1(a). The cluster is expected to move backward with a constant velocity $a<0$, without change in the shape. In other words, we assume a traveling wave solution $\rho(x, t)=\rho(X)$ and $v(x, t)=v(X)$ with $X=x-a t$. Following the procedures in $[33,28]$, we can show that the downstream front is a smooth transition layer and the upstream front is a shock wave.

3.1. The downstream and upstream fronts. For the downstream front that smoothly links a higher density region $\rho=\rho_{B}$ to a lower density region $\rho=\rho_{A}$, $\rho_{A}<\rho_{B}$, equations (1) and (2) are applicable. Hence, we replace $\rho(x, t)$ and $v(x, t)$ with $\rho(X)$ and $v(X)$ in the equations, which yields

$$
q=a \rho+q_{0}
$$

and

$$
\frac{d \rho}{d X}=g(\rho) \frac{q_{e}(\rho)-\left(a \rho+q_{0}\right)}{p^{\prime}(\rho) \rho^{2}-q_{0}}
$$

where the integration constant $q_{0}>0$, and thus the function $g(\rho)=\rho^{2}\left(\tau q_{0}\right)^{-1}>0$. Equation (3) suggests a linear relation between the flow $q$ and the density $\rho$, which 
is represented by a segment $\overline{A B}$ in Figure 1(b). As we assume that $d \rho /\left.d X\right|_{\rho=\rho_{A}}=$ $d \rho /\left.d X\right|_{\rho=\rho_{B}}=0$ (Figure 1(a)), (4) gives

$$
q_{e}\left(\rho_{A}\right)=a \rho_{A}+q_{0}, \quad q_{e}\left(\rho_{B}\right)=a \rho_{B}+q_{0} .
$$

This implies that the two constant states $\rho_{A}$ and $\rho_{B}$ are in equilibrium (see Figure 1(b)). See [23] for a similar derivation. Let $\left(\rho_{C}, q_{e}\left(\rho_{C}\right)\right)$ be the intersection of the segment $\overline{A B}$ and the fundamental diagram $q=q_{e}(\rho)$, i.e.,

$$
q_{e}\left(\rho_{C}\right)=a \rho_{C}+q_{0} .
$$

It is obvious that the numerator of (4) is positive for $\rho \in\left(\rho_{A}, \rho_{C}\right)$ and negative for $\rho \in\left(\rho_{C}, \rho_{B}\right)$ (see Figure $\left.1(\mathrm{~b})\right)$. Accordingly, a decreasing transition layer $(d \rho / d X<0)$ is guaranteed if and only if

$$
p^{\prime}\left(\rho_{C}\right) \rho_{C}^{2}-q_{0}=0, \quad\left(p^{\prime}(\rho) \rho^{2}-q_{0}\right)\left(\rho-\rho_{C}\right)>0 \quad \text { for } \rho \neq \rho_{C} .
$$

Intuitively, we have $q_{e}^{\prime}\left(\rho_{C}\right)<a<q_{e}^{\prime}\left(\rho_{B}\right)$; see Figure 1(b). This along with (5)-(7) gives $v_{e}^{\prime}\left(\rho_{C}\right)+p^{\prime}\left(\rho_{C}\right)<0$, and $v_{e}^{\prime}\left(\rho_{B}\right)+p^{\prime}\left(\rho_{B}\right)>0$. By the linear stability conditions (see section 3.2), the two inequalities imply the following property.

Property 1. For the solvability of a transition layer, (i) $\rho_{C}$ must be located in an unstable region, whereas (ii) $\rho_{B}$ must be located in a stable region.

The two formulations attained by choosing $p(\rho)=-v_{e}(\rho)$ in [27] and $p(\rho)=$ $-t_{r} v_{e}(\rho) / T$ in [26] (see Table 1) are not able to generate a transition layer, because equilibria of [27] and [26] can be easily shown to be uniformly stable and unstable, respectively. This also implies that they are not able to reproduce a wide cluster. For the choice of $p(\rho)=c_{0} \ln \rho$ in [11] (see Table 1), the detailed discussion on the solution of a wide cluster was given in [33]. These three models are excluded from the forthcoming discussion.

We also note that Siebel and Mauser $[19,20,21]$ adopted a strictly concave flowdensity relationship $q_{e}(\rho)=\rho v_{e}(\rho)$ with the "pressure" $p(\rho)=-v_{e}(\rho)$. Although the convective term of their models resembled the model in Zhang [27], Siebel and Mauser introduced a coefficient $\beta(\rho, v)$ in the relaxation term which may change sign to reflect the interaction or difference between the relaxation time and reaction time of drivers. This allowed two critical densities (and thus an unstable regime) which correspond to the roots of the function $\beta(\rho, v)$. Then a traveling wave solution was obtained for this novel formulation [20].

In another development, Greenberg [6] derived the traveling wave solutions (clusters) using Lagrangian coordinates, in which the headway $s=1 / \rho$ and the car index $m$ were taken as solution variables. Conservation across the discontinuous upstream front was also considered in [6], which in essence was similar to our discussion in what follows. Here, we remark that some intrinsic relations were implied in the formulations of Greenberg, Klar, and Rascle [8], Greenberg [6], and Siebel and Mauser [19].

In the forthcoming discussion, we assume that the denominator of (4) is an increasing function of $\rho$, that is,

$$
\left(p^{\prime}(\rho) \rho^{2}\right)^{\prime} \equiv \rho(\rho p(\rho))^{\prime \prime}>0,
$$

which is sufficient to ensure the inequality in (7). Essentially, (8) or the convexity of the function $\rho p(\rho)$ was also assumed in $[1,8,6,19,20,21]$. 
A monotonically increasing and smooth connection from $\rho_{A}$ to $\rho_{B}$ is impossible at the upstream front according to (3) and (4) and the detailed discussion in [33, 28]. We consider a shock wave with the following conservation form of (2),

$$
\frac{\partial \rho(v+p(\rho))}{\partial t}+\frac{\partial \rho v(v+p(\rho))}{\partial x}=\frac{q_{e}(\rho)-q}{\tau},
$$

in $[1,16]$. It is difficult, if not impossible, to define other conservation forms, except for that defined in [33] in which $c(\rho)=\rho p^{\prime}(\rho)$ was taken as a constant. We note that different conservation forms result in different values of the characteristic parameters for solving a wide cluster [28].

To deal with the assumed shock that is also a traveling wave with the moving speed $a<0$, we apply the Rankine-Hugoniot conditions to the conservation system of (1) and (9). This gives two equalities: one is implied in (5) and the other reads

$$
a=\frac{q_{e}\left(\rho_{B}\right)\left(v_{e}\left(\rho_{B}\right)+p\left(\rho_{B}\right)\right)-q_{e}\left(\rho_{A}\right)\left(v_{e}\left(\rho_{A}\right)+p\left(\rho_{A}\right)\right)}{\left(q_{e}\left(\rho_{B}\right)+\rho_{B} p\left(\rho_{B}\right)\right)-\left(q_{e}\left(\rho_{A}\right)+\rho_{A} p\left(\rho_{A}\right)\right)},
$$

where the constant state $\rho=\rho_{A}$ (together with $\rho=\rho_{B}$ ) can easily be verified to be in equilibrium by (1) and (9). By (5)-(7) and (10) we have five independent algebraic equations to solve for five unknowns: $a, \rho_{A}, \rho_{B}, \rho_{C}$, and $q_{0}$. The solution of this algebraic equation system determines the assumed wide cluster solution.

3.2. Control parameters and solvability of the wide cluster. The speeddensity relationship is taken as

$$
v_{e}(\rho)=v_{f}\left(\left(1+e^{12.5\left(\rho / \rho_{m}-0.25\right)}\right)^{-1}-\left(1+e^{12.5 \times 0.75}\right)^{-1}\right),
$$

where $v_{f}$ is the free-flow speed, $\rho_{m}$ is the jam density, and the point of inflexion of the fundamental diagram $q_{e}(\rho)=\rho v_{e}(\rho)$ is located at $\rho_{I} \approx 0.333598$. Equation (11) is similar to that in [12] and was also applied in [33]. We take the "pressure" as

$$
p(\rho)=\alpha\left(\rho / \rho_{m}\right)^{\gamma}, \quad \alpha, \gamma>0 .
$$

Let $\left(\rho_{0}, q_{e}\left(\rho_{0}\right)\right)$ be an equilibrium point in the fundamental diagram. The linear stability conditions for a constant solution $\rho=\rho_{0}$ can then be easily determined as $\lambda_{1}\left(\rho_{0}\right) \leq q_{e}^{\prime}\left(\rho_{0}\right) \leq \lambda_{2}\left(\rho_{0}\right)$, which implies that the kinematic wave speed lies between the first and second characteristic speeds. See Whitham [23] for the relevant discussion. The inequalities are equivalent to

$$
H\left(\rho_{0}\right) \equiv-1-\frac{v_{e}^{\prime}\left(\rho_{0}\right)}{p^{\prime}\left(\rho_{0}\right)} \leq 0,
$$

because it is assumed that $p^{\prime}\left(\rho_{0}\right)>0$, and the condition of a single point at the boundary with $\rho_{0}=0$ is excluded. Taking the equality of (13) and with two control parameters $\alpha$ and $\gamma$, we indicate the solvability of the critical densities and the resultant stable and unstable regions. Hereafter, we denote dimensionless variables by placing a bar over them, such that a density is scaled by $\rho_{m}$ and a speed (including the parameter $\alpha$ ) is scaled by $v_{f}$. Using the dimensionless variables, we note that these critical densities and all characteristic parameters of the cluster solution depend on $\gamma$ and $\bar{\alpha}$ only.

For the case $\gamma>1$, say $\gamma=2$ as is applied in the construction of Figure 2(a), monotone changes of the function $H\left(\rho_{0}\right)$ are divided into three intervals. This suggests 

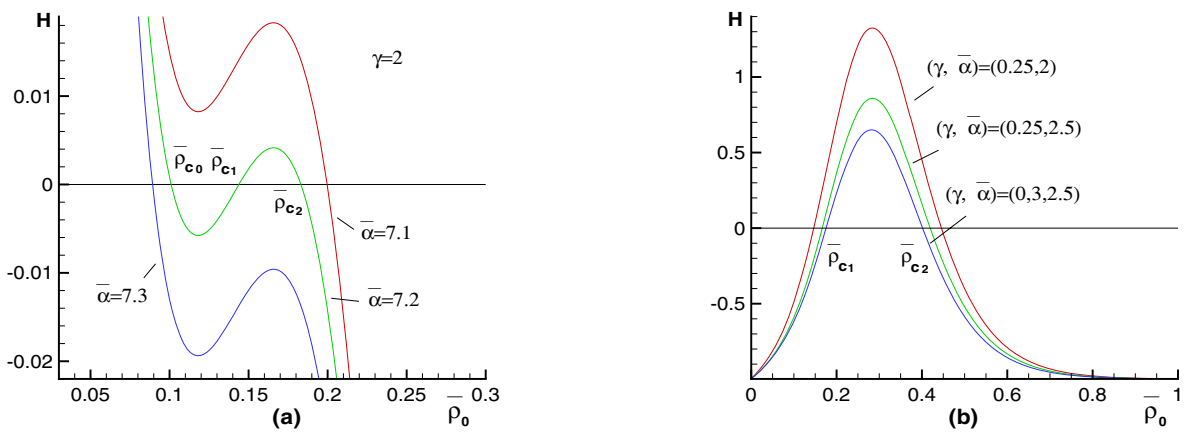

FIG. 2. Solvability of critical densities and division of stable and unstable regions for an equilibrium constant solution $\rho=\rho_{0}$ for (a) $\gamma>1$ and (b) $\gamma \leq 1$.

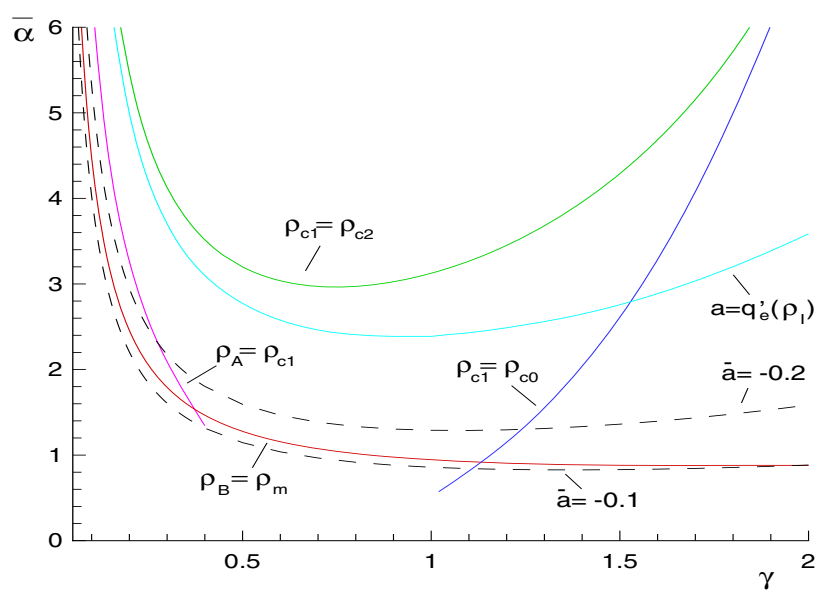

FIG. 3. Division of the $\gamma-\bar{\alpha}$ plane for the study of a wide cluster solution. The solution exists below the curve $a=q_{e}^{\prime}\left(\rho_{I}\right)$, and the traveling wave velocity a and the height $\rho_{B}-\rho_{A}$ decrease when $(\gamma, \bar{\alpha})$ approaches the curve. The equilibrium density $\rho=\rho_{A}$ of the cluster solution is located in a stable region only for approximately $\gamma<0.4$ and $(\gamma, \bar{\alpha})$ in or below the curve $\rho_{A}=\rho_{c_{1}}$.

at most three critical densities, $\rho_{c_{0}}, \rho_{c_{1}}$, and $\rho_{c_{2}}$. In this case, say with $\bar{\alpha}=7.2$ as in the figure, the three critical densities divide the interval $(0,1]$ into two stable intervals, $\left[\bar{\rho}_{c_{0}}, \bar{\rho}_{c_{1}}\right]$ and $\left[\bar{\rho}_{c_{2}}, 1\right]$, and two unstable intervals, $\left(0, \bar{\rho}_{c_{0}}\right)$ and $\left(\bar{\rho}_{c_{1}}, \bar{\rho}_{c_{2}}\right)$. As $\bar{\alpha}$ decreases (with reference to another curve $H\left(\bar{\rho}_{0}\right)$ for $\left.\bar{\alpha}=7.1<7.2\right), \bar{\rho}_{c_{1}}$ and $\bar{\rho}_{c_{0}}$ become identical for some $\bar{\alpha} \in(7.1,7.2)$. This is to simultaneously have $H\left(\rho_{c_{0}}\right)=0$ and $H^{\prime}\left(\rho_{c_{0}}\right)=0$, which determine a curve that is denoted by $\rho_{c_{1}}=\rho_{c_{0}}$ in the $\gamma-\bar{\alpha}$ coordinate plane (Figure 3). In the region that is below the curve $\rho_{c_{1}}=\rho_{c_{0}}$ (Figure 3), the critical densities $\bar{\rho}_{c_{0}}$ and $\bar{\rho}_{c_{1}}$ together with the interval $\left[\bar{\rho}_{c_{0}}, \bar{\rho}_{c_{1}}\right]$ must vanish because $\bar{\alpha}$ becomes smaller; see also the reference curve $H\left(\bar{\rho}_{0}\right)$ for $\bar{\alpha}=7.1<7.2$ in Figure 2(a). In this case, we have an unstable interval $\left(0, \bar{\rho}_{c_{2}}\right)$ and a stable interval $\left[\bar{\rho}_{c_{2}}, 1\right]$. Similarly, we can draw a curve $\rho_{c_{1}}=\rho_{c_{2}}$ in Figure 3 , which is determined by setting $H\left(\rho_{c_{2}}\right)=0$ and $H^{\prime}\left(\rho_{c_{2}}\right)=0$. The critical densities $\bar{\rho}_{c_{1}}$ and $\bar{\rho}_{c_{2}}$ along with the interval $\left(\bar{\rho}_{c_{1}}, \bar{\rho}_{c_{2}}\right)$ vanish for $(\gamma, \bar{\alpha})$ in the region that is above this curve (Figure 3$)$. See also the reference curve $H\left(\bar{\rho}_{0}\right)$ for $\bar{\alpha}=7.3>7.2$ in Figure $2(\mathrm{a})$. In this case, we have an unstable interval $\left(0, \bar{\rho}_{c_{0}}\right)$ and a stable interval $\left[\bar{\rho}_{c_{0}}, 1\right]$. 
For the case $\bar{\gamma} \leq 1$, monotone changes of the function $H\left(\rho_{0}\right)$ are divided into two intervals. This suggests at most two critical densities $\bar{\rho}_{c_{1}}$ and $\bar{\rho}_{c_{2}}$. We define the same curve $\rho_{c_{1}}=\rho_{c_{2}}$ as previously discussed, and the curve goes smoothly in the whole region for $\gamma>1$ and $\gamma \leq 1$ (Figure 3). However, in the region that is above the curve $\rho_{c_{1}}=\rho_{c_{2}}$ and for $\gamma \leq 1$, we have an overall stable interval $(0,1]$.

We now turn our attention to the solvability and the characteristic parameters of the discussed wide cluster, which is related to the critical densities and the division of the stable and unstable intervals through Property 1 and the following discussion. With two control parameters $\bar{\alpha}$ and $\gamma$, we note that one equation in addition to (5)-(7) and (10) determines a curve in the $\gamma-\bar{\alpha}$ coordinate plane implicitly, which is also shown in Figure 3.

Defining the curve $\rho_{B}=\rho_{m}$, we can verify that the region below the curve $\rho_{B}=\rho_{m}$ suggests that $\rho_{B}>\rho_{m}$, by which the wide cluster solution is not collisionfree. Therefore, this region is not considered in the forthcoming discussion. Furthermore, we define the curve $a=q_{e}^{\prime}\left(\rho_{I}\right)\left(\approx-0.542579 v_{f}\right)$. By Figure 1(b), it is evident that the traveling wave speed $a$ reaches its minimum with $\rho_{A}=\rho_{C}=\rho_{B}=\rho_{I}$ for $(\gamma, \bar{\alpha})$ in this curve. Here, the inequalities

$$
\rho_{A}<\min \left(\rho_{C}, \rho_{I}\right)<\rho_{B}
$$

are assumed for the wide cluster solution because of the nonconvexity of the function $q_{e}(\rho)$ (cf. Figure 1(b)). According to Property 1, it is implied that $\rho_{I}$ is a critical density in the above limiting solution. Actually, we derive the same curve when defining $\rho_{c_{2}}=\rho_{I}$. For $(\gamma, \bar{\alpha})$ that is above this curve $\left(a=q_{e}^{\prime}\left(\rho_{I}\right)\right.$ or $\left.\rho_{c_{2}}=\rho_{I}\right),(5)-(7)$ and (10) are insolvable. This means that the iteration that is applied to solve these equations is never convergent under the restriction of (14). On the other hand, (5)-(7) and (10) are solvable for $(\gamma, \bar{\alpha})$ that is between the curves $\rho_{B}=\rho_{m}$ and $a=q_{e}^{\prime}\left(\rho_{I}\right)$. Here, the curve $a=q_{e}^{\prime}\left(\rho_{I}\right)$ serves as the other boundary to separate the two regions in which the wide cluster is, respectively, solvable and insolvable.

Let $\alpha$ increase. Then we find that the traveling wave speed $a$ of the wide cluster decreases until $a$ reaches its limiting value in the curve $a=q_{e}^{\prime}\left(\rho_{I}\right)$. Two reference curves $\bar{a}=-0.1$ and $\bar{a}=-0.2$ are depicted in Figure 3 to show such a monotonic decreasing property. By the mass conservation at the discontinuous upstream front, a decreasing traveling wave speed usually suggests a drastic drop in the height $\rho_{B}-\rho_{A}$ of the wide cluster. The critical density $\rho_{c_{2}}$ also decreases in this trend, which implies that $\rho_{c_{2}}>\rho_{I}$ for $(\gamma, \bar{\alpha})$ below the curve $a=q_{e}^{\prime}\left(\rho_{I}\right)$, and $\rho_{c_{2}}<\rho_{I}$ for $(\gamma, \bar{\alpha})$ between the curves $a=q_{e}^{\prime}\left(\rho_{I}\right)$ and $\rho_{c_{1}}=\rho_{c_{2}}$. This seems to suggest that it is essential that there exist a critical density that is greater than the inflexion $\rho_{I}$ for the solvability of the wide cluster. On the other hand, it is novel that the wide cluster is insolvable even though we do have a critical density $\rho_{c_{2}}<\rho_{I}$ that is close to $\rho_{I}$.

The foregoing discussion together with Property 1 also suggests the locational relations between the critical densities and the two characteristic densities $\rho_{C}$ and $\rho_{B}$. That is, $\rho=\rho_{C}$ and $\rho=\rho_{B}$ (as a constant portion of the wide cluster), respectively, are located in two adjacent unstable and stable intervals that is separated by $\rho_{c_{2}}>\rho_{I}$. This fact is also related to the following analytical property.

Property 2. There is at most one critical density in $\left[\rho_{I}, \rho_{m}\right]$.

This property holds simply because a critical density is also the root of the function $\rho^{2} p^{\prime}(\rho) H(\rho)$, and $\left(\rho^{2} p^{\prime}(\rho) H(\rho)\right)^{\prime}=-\rho(\rho p(\rho))^{\prime \prime}-\rho\left(\rho v_{e}(\rho)\right)^{\prime \prime}<0$ in $\left[\rho_{I}, \rho_{m}\right]$. For the solvability of the wide cluster, we do have such a critical density $\rho_{c_{2}}$. Here, it is implied that $\rho_{c_{0}} \leq \rho_{c_{1}}<\rho_{I}$ if the critical density $\rho_{c_{0}}$ or $\rho_{c_{1}}$ does exist. 
To learn the locational relation between $\rho_{A}$ and the critical densities, we draw the curve $\rho_{A}=\rho_{c_{1}}$ in Figure 3, which exists for $\gamma \leq 0.4$ approximately, where it meets with the curve $\rho_{B}=\rho_{m}$. Between the two curves we have $\rho_{A} \leq \rho_{c_{1}}$, which suggests that the equilibrium $\rho=\rho_{A}$ (as a constant portion of the wide cluster) is stable. In other regions where $\rho_{c_{1}}$ does not vanish, we obviously have $\rho_{c_{1}}<\rho_{A}<\rho_{I}<\rho_{c_{2}}$, which suggests that $\rho=\rho_{A}$ is unstable. In the region that is below the curve $\rho_{c_{0}}=\rho_{c_{1}}$ for $\gamma>1$, where $\rho_{c_{0}}$ and $\rho_{c_{1}}$ vanish, $\rho=\rho_{A}<\rho_{I}<\rho_{c_{2}}$ still belongs to the unstable region $\left(0, \rho_{c_{2}}\right)$.

4. Wide clusters derived from numerical simulation. We write the system of (1) and (9) as the following standard conservation or balance laws:

$$
u_{t}+f(u)_{x}=s(u)
$$

where $u=(\rho, h)^{T}, h=\rho(v+p(\rho)), f(u)=\left(h-\rho p(\rho), \rho^{-1} h^{2}-h p(\rho)\right)^{T}$, and $s(u)=$ $\left(0, \tau^{-1}\left(q_{e}(\rho)-h+\rho p(\rho)\right)\right)^{T}$. For a numerical simulation, a conservative scheme of system (15) can be written as

$$
u_{i}^{n+1}=u_{i}^{n}-\frac{\Delta t^{n}}{\Delta x}\left(\hat{f}_{i+1 / 2}^{n}-\hat{f}_{i-1 / 2}^{n}\right)+\Delta t^{n} s\left(u_{i}^{n}\right),
$$

where $\Delta x=L / N$, and $N$ is the grid number of the computational interval $(0, L)$. We apply the Lax-Friedrichs numerical flux:

$$
\hat{f}_{i+1 / 2}^{n}=\frac{1}{2}\left(f\left(u_{i}^{n}\right)+f\left(u_{i+1}^{n}\right)-\mu^{n}\left(u_{i+1}^{n}-u_{i}^{n}\right)\right), \quad \mu^{n}=\max _{u} \max \left(\left|\lambda_{1}\right|,\left|\lambda_{2}\right|\right),
$$

where $\lambda_{1,2}$ are the two characteristic speeds, and the maximum is taken over $u_{i}^{n}$ for all $i$ at time level $n$. The CFL condition that is necessary for numerical stability turns out to be $\Delta t^{n} \leq \mu^{n} \Delta x$; we always take $\Delta t^{n}=0.7 \mu^{n} \Delta x$ in numerical simulations. As the grids should be sufficiently refined to obtain the discussed wide cluster solution, we take a large grid number $N=10000$ in all examples. For illustration purposes, only one state in every 20 grid points is shown in the figures. See [18, 22] for detailed accounts of the Lax-Friedrichs scheme. The reason for the application of this scheme rather than the Godunov or a higher-order scheme was explained in [28].

For an initial constant distribution $\rho(x, 0)=\rho_{0}$, which is in equilibrium with $v(x, 0)=v_{e}(\rho(x, 0))$, the small perturbation

$$
\rho(x, 0)=\rho_{0}+0.005 \rho_{0}(\operatorname{sgn}(0.05-|x / L-0.5|)+1) \sin (20 \pi(x / L-0.5))
$$

induces amplifying oscillations if density $\rho_{0}$ is located in an unstable region. Here, we define $\operatorname{sgn}(s)=1$ for $s \geq 0$, and $\operatorname{sgn}(s)=-1$ for $s<0$; the integral average density of $\rho(x, 0)$ over the computational interval $[0, L]$ is not changed by the perturbation. Applying the periodic boundary conditions

$$
\rho(0, t)=\rho(L, 0), \quad v(0, t)=v(L, 0)
$$

which ensure the conservation of the total vehicles in numerical simulation, the oscillations may evolve into stop-and-go waves or wide clusters in the long run if $\rho_{0}$ is sufficiently large. The dynamics of the evolution was well described in the PW [15, 23], Kühne [13], and KK [12] models; see also [28] for more relevant discussion.

As discussed in previous sections, the evolution of the perturbed constant flow $\rho=\rho_{0}$ is dependent on the control parameters $\gamma$ and $\bar{\alpha}$, as shown in Figure 3. Our 


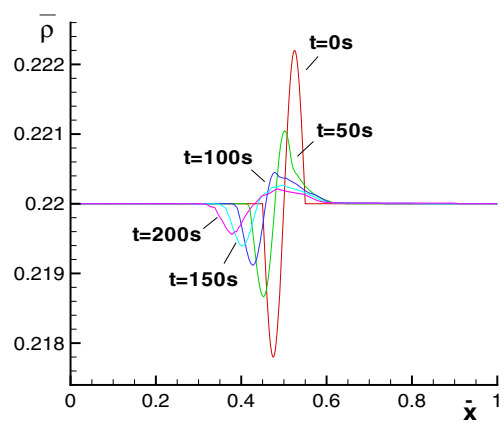

(a)

(c)

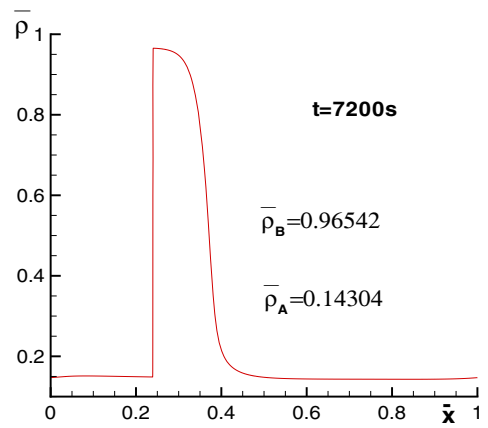

FIG. 4. Evolution of unstable equilibrium flow $\rho=\rho_{0}$ with small perturbation for $(\gamma, \bar{\alpha})$ in different domains in Figure 3. (a) $(\gamma, \bar{\alpha})=(0.3,4.2), \bar{\rho}_{0}=0.22$, and $\tau=10$ s; (b) $(\gamma, \bar{\alpha})=(0.7,2.8)$, $\bar{\rho}_{0}=0.25$, and $\tau=10 \mathrm{~s}$; (c) $(\gamma, \bar{\alpha})=(0.25,2.1), \bar{\rho}_{0}=0.25$, and $\tau=18 \mathrm{~s} ;$ (d) $(\gamma, \bar{\alpha})=(0.5,1.5)$, $\bar{\rho}_{0}=0.32$, and $\tau=10 \mathrm{~s}$.

TABLE 2

Characteristic parameters of a wide cluster and critical densities for comparison to the numerical results in Figures 4 and 5, where "IS" means insolvable and the values in the bracket are obtained from numerical simulation.

\begin{tabular}{|c|c|c|c|c|c|c|c|c|}
\hline$\gamma$ & $\bar{\alpha}$ & $\bar{\rho}_{A}$ & $\bar{\rho}_{B}$ & $\bar{\rho}_{C}$ & $\bar{a}$ & $\bar{\rho}_{c 0}$ & $\bar{\rho}_{c 1}$ & $\bar{\rho}_{c 2}$ \\
\hline 0.3 & 4.2 & IS & IS & IS & IS & IS & IS & IS \\
\hline 0.7 & 2.8 & IS & IS & IS & IS & IS & 0.226662 & 0.303168 \\
\hline 0.25 & 2.1 & 0.142860 & 0.968573 & 0.332912 & -0.137028 & IS & 0.150555 & 0.440170 \\
& & $(0.14304)$ & $(0.96542)$ & & & & & \\
\hline 0.5 & 1.5 & 0.153584 & 0.817781 & 0.334882 & -0.176989 & IS & 0.139590 & 0.423337 \\
& & $(0.15439)$ & $(0.81369)$ & & & & & \\
\hline 1.5 & 1.5 & 0.162911 & 0.680572 & 0.346706 & -0.229506 & IS & IS & 0.401206 \\
& & $(0.16368)$ & $(0.67614)$ & & & & & \\
\hline
\end{tabular}

numerical tests agree well with all these descriptions. In the domain that is on or above the curve $\rho_{c_{1}}=\rho_{c_{2}}$ in Figure 3, the perturbation decays with time because the solution $\rho=\rho_{0}$ is stable if $\bar{\gamma} \leq 1$ or, otherwise, if $\rho_{0}$ is greater than $\rho_{c_{0}}$, which is usually very small (see Figure $4(\mathrm{a})$ ). In the domain between the curves $\rho_{c_{1}}=\rho_{c_{2}}$ and $a=q_{e}^{\prime}\left(\rho_{I}\right)$, the perturbation increases with time but a wide cluster solution can never be developed regardless of the length of simulation (see Figure 4(b)). Here and hereafter, for all figures, the related parameters are shown in Table 2 and the variable $x$ is scaled by $\bar{x}=x / L$. Through numerical simulation, we can always derive one or more wide clusters in other domains where the solution is predicted analytically. Two 


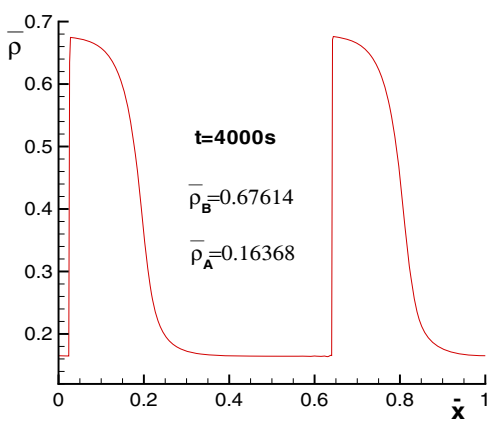

(a)

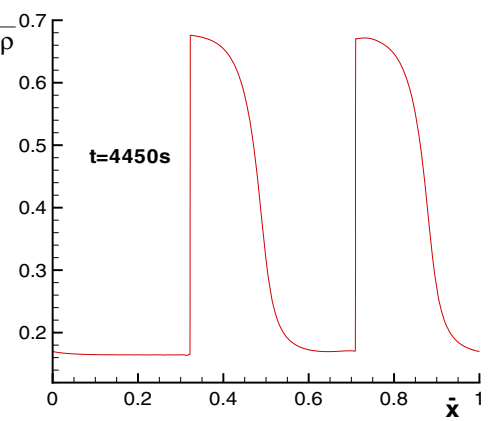

(b)
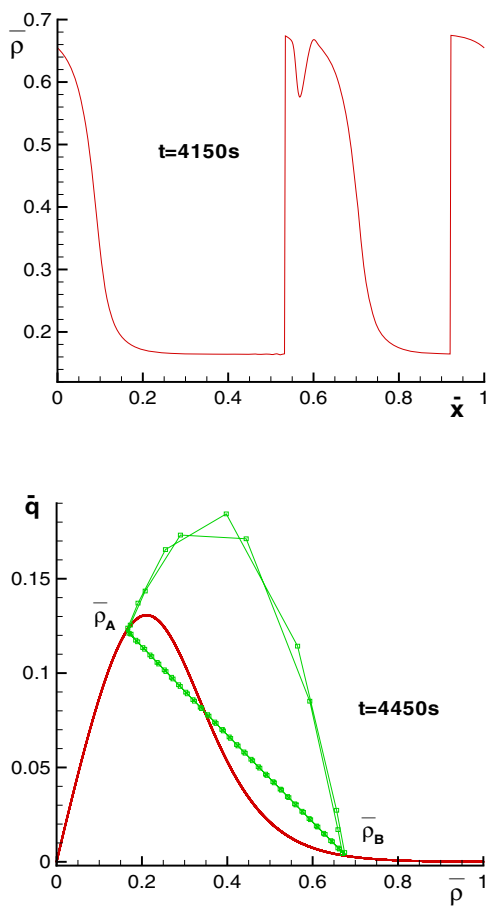

(d)

FIG. 5. Stability test of a wide cluster solution with $(\gamma, \bar{\alpha})=(1.5,1.5), \bar{\rho}_{0}=0.33$, and $\tau=10 \mathrm{~s}$ : (a) two fully developed clusters; (b) density change due to a perturbation to the cluster solution; (c) recovery of the cluster solution from the perturbation; (d) the fundamental diagram $\left(\bar{\rho}, \bar{q}_{e}(\bar{\rho})\right)$ and the phase plot $(\bar{\rho}, \bar{q})$.

such examples are shown in Figures 4(c) and (d).

It is novel that all predicted wide clusters are stable through numerical testing, even though the density $\rho=\rho_{A}$ of the free traffic is located in an unstable region. It is evident that a stable wide cluster solution exists with one or three critical densities for $\bar{\gamma}>1$, as predicted analytically in Figure 3. We show a stability test in Figure 5 with $(\bar{\gamma}, \alpha)$ in this region, in which there is only one critical density (see also Table 2). Figure 5(a) shows two wide clusters that are derived at $t=4000 \mathrm{~s}$. By a perturbation that changes the speed $\bar{v}$ to $\bar{v} \mp 0.1$ for $\bar{x}$ between 0.5 and $0.5 \mp 0.01$, one cluster is found to be slightly distorted at $t=4150 \mathrm{~s}$. However, it soon recovers, as shown in Figure 5(c). In comparison to the fundamental diagram, we show the phase plot of the solution in Figure 5(d), where the segment $\overline{A B}$ represents the acceleration path of the downstream front. Nevertheless, the deceleration path of the two upstream fronts is now replaced by two curves from $A$ to $B$. This takes place because a shock profile has to be smoothed by numerical viscosities, which can hardly be avoided in any scheme. The clusters that are shown in all of these figures follow the pattern that is anticipated in Figures 1(a) and (b).

A stable wide cluster solution with a length of unstable equilibrium $\rho=\rho_{A}$ might be well explained by the stable structure of the upstream front. Actually, the relaxation term of (9) vanishes for solution states on the both sides near the upstream front. Moreover, it is easy to verify that the Lax entropy conditions are satisfied with respect to this discontinuity (see also [6]). When the periodic boundary 
conditions are applied, a perturbation to $\rho=\rho_{A}$ (or other solution states) of the wide cluster is expected to be overtaken and thus "absorbed" by the upstream-moving shock. However, an affirmative conclusion could be made only through a rigorous mathematical proof, which is an interesting question for future study.

The parameter values of $\rho_{A}$ and $\rho_{B}$ acquired from numerical simulations are also shown in the figures, which are in good agreement with those that are derived from (5)-(7) and (10), and are listed in Table 2. This demonstrates that our numerical solutions converge to the analytical solutions of the described wide cluster.

5. Conclusions and discussions. We have thoroughly investigated the admissibility of a wide cluster solution in "anisotropic" higher-order models, in which the acceleration equation takes the functional form in (2). By this functional form (and also those in [6] and [19]), it appears essential that for the existence of the wide cluster solution the speed-density relationship $v_{e}(\rho)$ and the "pressure" $p(\rho)$ should be in "conflict" such that there exists an unstable regime in the vicinity of the congestion. Accordingly, it is evident that some formulations that are mentioned in this paper (and probably others) do not admit any wide cluster solution. Even with the functional forms that allow an unstable regime, the "pressure" $p(\rho)$ and the speed-density relationship $v_{e}(\rho)$ that represents the "force" for relaxation or fluctuation should act "harmoniously" to admit the solution. With reference to Figure 3, this means that a wide cluster is not always ensured or physically sound for all combinations of control parameters $(\gamma, \bar{\alpha})$. The intrinsic relationships between the aforementioned functions or "forces" can be left for future study.

\section{REFERENCES}

[1] A. Aw And M. Rascle, Resurrection of "second order" models of traffic flow, SIAM J. Appl. Math., 60 (2000), pp. 916-938.

[2] A. Aw, A. Klar, T. Materne, And M. Rascle, Derivation of continuum traffic flow models from microscopic follow-the-leader models, SIAM J. Appl. Math., 63 (2002), pp. 259-278.

[3] P. Bagnerini and M. Rascle, A multiclass homogenized hyperbolic model of traffic flow, SIAM J. Math. Anal., 35 (2003), pp. 949-973.

[4] C. F. Daganzo, A behavioral theory of multi-lane traffic flow, part I: Long homogeneous freeway sections, Transportation Res. B, 36 (2002), pp. 131-158.

[5] C. F. Daganzo, Requiem for second-order fluid approximations of traffic flow, Transportation Res. B, 29 (1995), pp. 277-286.

[6] J. M. Greenberg, Congestion redux, SIAM J. Appl. Math., 64 (2004), pp. 1175-1185.

[7] J. M. Greenberg, Extensions and amplifications of a traffic flow model of Aw and Rascle, SIAM J. Appl. Math., 62 (2001), pp. 729-745.

[8] J. M. Greenberg, A. Klar, and M. Rascle, Congestion on multilane highways, SiAM J. Appl. Math., 63 (2003), pp. 818-833.

[9] A. K. Gupta and V. K. KatiYar, A new multi-class continuum model for traffic flow, Transportmetrica, 3 (2007), pp. 73-85.

[10] M. Herty, C. Kirchener, And S. Moutari, Multi-class traffic models on road networks, Commun. Math. Sci., 4 (2006), pp. 591-608.

[11] R. JiAng, Q. S. WU, AND Z. J. ZhU, A new continuum model for traffic flow and numerical tests, Transportation Res. B, 36 (2002), pp. 405-419.

[12] B. S. Kerner And P. KonhäUser, Structure and parameters of clusters in traffic flow, Phys. Rev. E (3), 50 (1994), pp. 54-83.

[13] R. D. KüHne, Macroscopic freeway model for dense traffic-stop-start waves and incident detection, in Proceedings of the 9th International Symposium on Transportation and Traffic Theory, J. Volmuller and R. Hamerslag, eds., VNU Science Press, Utrecht, 1984, pp. 21-42.

[14] M. J. Lighthill and G. B. Whitham, On kinematic waves: II A theory of traffic flow on long crowded roads, Proc. Roy. Soc. London Ser. A, 229 (1955), pp. 317-345.

[15] H. J. Payne, Models of freeway traffic and control, in Mathematical Models of Public Systems, Vol. 1, A. G. Bekey, ed., Simulation Council Proc. Ser. 1, La Jolla, CA, 1971, pp. 51-61.

Copyright $@$ by SIAM. Unauthorized reproduction of this article is prohibited. 
[16] M. RAscle, An improved macroscopic model of traffic flow: Derivation and links with the Lighthill-Whitham model, Math. Comput. Modelling, 35 (2002), pp. 581-590.

[17] P. I. Richards, Shock waves on the highway, Oper. Res., 4 (1956), pp. 42-51.

[18] C.-W. SHU, Essentially non-oscillatory and weighted essentially non-oscillatory schemes for hyperbolic conservation laws, in Advanced Numerical Approximation of Nonlinear Hyperbolic Equations, B. Cockburn, C. Johnson, C.-W. Shu, and E. Tadmor, Lecture Notes in Math. 1697, A. Quarteroni, ed., Springer, Berlin, 1998, pp. 325-432.

[19] F. Siebel And W. Mauser, On the fundamental diagram of traffic flow, SIAM J. Appl. Math., 66 (2006), pp. 1150-1162.

[20] F. Siebel AND W. Mauser, Synchronized flow and wide moving jams from balanced vehicular traffic, Phys. Rev. E (3), 73 (2006), article 066108.

[21] F. SieBel AND W. Mauser, Balanced vehicular traffic at a bottleneck, Phys. A, submitted.

[22] E. F. Toro, Riemann Solvers and Numerical Methods for Fluid Dynamics, Springer-Verlag, Berlin, 1999.

[23] G. B. Whitham, Linear and Nonlinear Waves, John Wiley and Sons, New York, 1974.

[24] R. E. Wilson AND P. BERG, Existence and classification of traveling wave solutions to second order highway traffic models, in Traffic and Granular Flow '01, M. Fukui, Y. Sugiyama, M. Schreckenberg, and D. E. Wolf, eds., Springer, Berlin, 2003, pp. 85-90.

[25] G. C. K. Wong And S. C. Wong, A multi-class traffic flow model-an extension of LWR model with heterogeneous drivers, Transportation Res. A, 36 (2002), pp. 827-841.

[26] Y. Xue And S. Q. DaI, Continuum traffic model with the consideration of two delay time scales, Phys. Rev. E (3), 68 (2003), article 066123.

[27] H. M. Zhang, A non-equilibrium traffic model devoid of gas-like behavior, Transportation Res. B, 36 (2002), pp. 275-290.

[28] P. Zhang And S. C. Wong, Essence of conservation forms in the traveling wave solutions of higher-order traffic flow models, Phys. Rev. E (3), 74 (2006), article 026109.

[29] P. Zhang, R. X. LiU, And S. C. Wong, High-resolution numerical approximation of traffic flow problems with variable lanes and free-flow velocities, Phys. Rev. E (3), 71 (2005), article 056704 .

[30] P. Zhang, R. X. LiU, S. C. Wong, And S. Q. Dai, Hyperbolicity and kinematic waves of a class of multi-population partial differential equations, European J. Appl. Math., 17 (2006), pp. 171-200.

[31] M. P. Zhang, C. W. Shu, G. C. K. Wong, and S. C. Wong, A weighted essentially nonoscillatory numerical scheme for a multi-class Lighthill-Whitham-Richards traffic flow model, J. Comput. Phys., 191 (2003), pp. 639-659.

[32] P. Zhang, S. C. Wong, and C. W. Shu, A weighted essentially non-oscillatory numerical scheme for a multi-class traffic flow model on an inhomogeneous highway, J. Comput. Phys., 212 (2006), pp. 739-756.

[33] P. Zhang, S. C. Wong, And S. Q. DAI, Characteristic parameters of a wide cluster in a higher-order traffic flow model, Chinese Phys. Lett., 23 (2006), pp. 516-519.

Copyright $@$ by SIAM. Unauthorized reproduction of this article is prohibited. 